1. The Effect of Short Pulse Width Settings on the Therapeutic Window in Subthalamic Nucleus Deep Brain Stimulation for Parkinson's disease

2. Viswas Dayal ${ }^{1}$, Timothy Grover ${ }^{2}$, Patricia Limousin ${ }^{3}$, Joseph Candelario, Maricel Salazar, Elina Tripoliti, Ludvic Zrinzo, Jonathan Hyam, Marjan Jahanshahi, Marwan Hariz, Thomas Foltynie ${ }^{4}$

3. ${ }^{1}$ Sobell Department of Motor Neuroscience, UCL Institute of Neurology \& The National Hospital for Neurology and Neurosurgery, Queen Square, London, WC1N 3BG, United Kingdom. Electronic address: v.dayal@ucl.ac.uk

2Sobell Department of Motor Neuroscience, UCL Institute of Neurology \& The National Hospital for Neurology and Neurosurgery, Queen Square, London, WC1N 3BG, United Kingdom. Electronic address: Tim.Grover@uclh.nhs.uk

${ }^{3}$ Sobell Department of Motor Neuroscience, UCL Institute of Neurology \& The National Hospital for Neurology and Neurosurgery, Queen Square, London, WC1N 3BG, United Kingdom. Electronic address: p.limousin@ucl.ac.uk

${ }^{4}$ Sobell Department of Motor Neuroscience, UCL Institute of Neurology \& The National Hospital for Neurology and Neurosurgery, Queen Square, London, WC1N 3BG, United Kingdom. Electronic address: t.foltynie@ucl.ac.uk

4. [Same as above]

5. Short Pulse Width DBS in Parkinson's disease

6. Correspondence:

Dr Viswas Dayal

Sobell Department of Motor Neuroscience, UCL Institute of Neurology \& The National Hospital for Neurology and Neurosurgery, Box 146, Queen Square, London, WC1N 3BG.

Email address: v.dayal@ucl.ac.uk

Telephone number: +44(0)203 4488736

Fax number: $+44(0) 2034488642$ 


\section{The Effect of Short Pulse Width Settings on the Therapeutic Window in Subthalamic Nucleus Deep Brain Stimulation for Parkinson's disease}

Viswas Dayal, Timothy Grover, Patricia Limousin, Joseph Candelario, Maricel Salazar, Elina

Tripoliti, Ludvic Zrinzo, Jonathan Hyam, Marjan Jahanshahi, Marwan Hariz, Thomas Foltynie

\section{ABSTRACT}

\section{BACKGROUND:}

Subthalamic nucleus deep brain stimulation (STN-DBS) is an established treatment for selected Parkinson's disease (PD) patients, but therapy is often limited by side effects. Previous studies indicate an inverse relationship of the therapeutic window (TW) to pulse width (PW) settings down to $60 \mu \mathrm{s}$, but there is limited data available on the effect of shorter PWs.

\section{OBJECTIVES:}

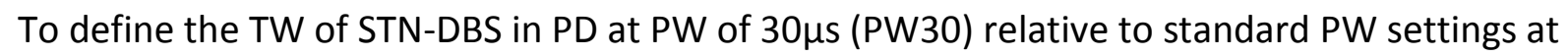
$60 \mu \mathrm{s}$ (PW60), and to compare speed of gait and intelligibility of speech on the two PW conditions.

\section{METHODS:}

Monopolar review data of 15 consecutive PD patients who had screening of contacts performed at PW60 and PW30 was used to calculate the TWs on each setting. We compared the TWs of the most efficacious contact per STN, and a secondary analysis was performed comparing all contacts. Speed of gait with timed 10 metre walk test and intelligibility of speech were also compared at the efficacy thresholds for PW60 and PW30.

\section{RESULTS:}

The TW was significantly greater at PW30 $[3.8 \pm 1.6 \mathrm{~mA}]$ than at PW60 $[1.7 \pm 1.1 \mathrm{~mA}]$. In the secondary analysis, 110 TWs could be calculated and these remained significantly higher at PW30. The timed 10 metre walk at PW30 was faster than at PW60, and the mean speech intelligibility assessment scores were significantly improved at PW30.

\section{CONCLUSIONS}

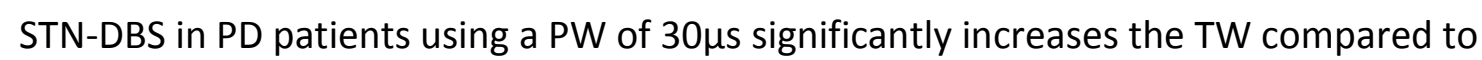
standard PW settings, and this effect is consistent across all contacts of an electrode. Speed of gait and speech intelligibility are also improved at $30 \mu$ s settings.

\section{KEYWORDS:}


Deep Brain Simulation Deep brain stimulation (DBS), Parkinson's Disease (PD), Subthalamic

Nucleus (STN), Electrical Parameters, Pulse Width, Side Effects, Speech Intelligibility, Gait.

\section{INTRODUCTION}

Deep brain stimulation (DBS) of the subthalamic nucleus (STN) is a well-established treatment option for patients with Parkinson's disease (PD) with motor fluctuations and dyskinesias refractory to medical therapy (1-4). However, the use of conventional programming parameters presents limitations in some patients due to the emergence of side effects at levels of stimulation required to counteract Parkinsonian motor symptoms (59).

The stimulation intensity, represented by current or voltage, is the usual parameter titrated to adjust the level of stimulation. This has led to stimulation intensity being used as the basis for defining the therapeutic window (TW), which is the magnitude of difference between the threshold for the required therapeutic effect and the threshold for side effects. Early studies examining the effect of pulse width (PW) between 60 and $450 \mu$ s on therapeutic and adverse effects in PD patients noted wider therapeutic windows at lower PWs. The lowest available PW of $60 \mu$ s on conventional systems was established as the standard value to be used in STN-DBS for optimal symptom control while minimising side effects in PD patients over the last two decades (10-13).

With technological advances in the last few years, it has become possible to utilise pulse width settings of less than $60 \mu$ s with systems produced by selected manufacturers. Moreover, there are suggestions from modelling data that various PWs may selectively stimulate different neural elements $(14,15)$. A wider TW than currently exists may provide more scope to increase stimulation both initially and over time without the occurrence of adverse effects at the level required for optimal control of PD motor symptoms.

There has, however, been little published data looking at whether the effect of increasing

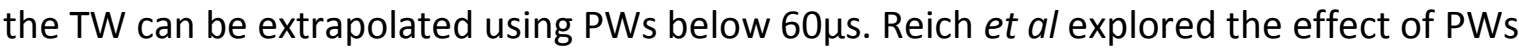
between 10 and $120 \mu$ s on the therapeutic window in four patients and reported an increase TW of $182 \%$ at $30 \mu$ s relative to $60 \mu \mathrm{s}$. They also noted that despite using a higher stimulation current at lower PWs, the energy used in terms of charge per pulse was actually lower than at $60 \mu \mathrm{s}$. However, efficacy thresholds could not be determined in many patients at PWs below $30 \mu$ s due to the need for very high stimulation current (14).

Here, we report our findings on the effect of short PW on the therapeutic window from a larger series of patients in the early post-operative setting. Our retrospective analysis of monopolar review data aimed to quantitatively define the therapeutic window at a PW of $30 \mu$ s relative to standard PW $(60 \mu \mathrm{s})$ in patients with STN stimulation for PD. In addition to this, we compare speed of gait, intelligibility of speech, and total electrical energy delivered 
(TEED) between standard (PW60) and short (PW30) pulse width settings at the thresholds for maximal rigidity control.

\section{PATIENTS AND METHODS}

Fifteen consecutive PD patients ( 7 female, mean age $55 \pm 8$ years) who underwent DBS surgery with bilateral implantation of electrodes in the subthalamic nucleus using Boston Scientific Vercise and Vercise PC systems in the 12 month period through to October 2017 were included. The subjects underwent an extended monopolar review 2-10 days postoperatively (mean $4.3 \pm 2.5$ ) as part of standard clinical assessment to screen for therapeutic and adverse effects of stimulation at each contact prior to initiating DBS therapy.

All patients were assessed in the OFF medication state, having withheld all dopaminergic medication at least 12 hours prior to screening. Each of the four contacts on each side was screened individually to determine the efficacy threshold and the side effect threshold to the nearest $0.1 \mathrm{~mA}$, according to our usual standard of care. We defined the efficacy threshold as the lowest current required to produce complete or near complete loss of contralateral rigidity. Rigidity was assessed at the wrist, initially with $0.5 \mathrm{~mA}$ increments of stimulation current at 60 second intervals until maximal loss of rigidity was achieved. The exact threshold was then determined with assessments at $0.1 \mathrm{~mA}$ increments.

The same procedure was followed to determine the side effect threshold, which was defined as the first clinically evident side effect reported by the patient or observed by the assessing clinician that either persisted for greater than two minutes or was not tolerated by the patient for this length of time.

Assessments were done at standard pulse width $(60 \mu \mathrm{s})$ then repeated at short pulse width $(30 \mu \mathrm{s})$ with a one hour interval between sessions. The frequency setting was kept constant at $130 \mathrm{~Hz}$ for all patients, and the IPG was always programmed as anode. Stimulation was programmed in ring mode only for each contact. The therapeutic window was calculated as the difference between the side effect and efficacy thresholds for each of these contacts at standard and short PW. Speed of gait was assessed with a timed 10 metre walk at the efficacy threshold for each PW condition.

We performed two sets of analyses for the therapeutic window: First, we compared the most efficacious contact (i.e. the one with the lowest efficacy threshold at 60 $\mu$ s) in each STN electrode with repeated measures on the two PW conditions using a paired sample t-test. A further comparison was then made with paired samples for the entire set of contacts (four per STN) on the two PW conditions, to determine if any change in TW was consistent across alternative contacts to those used in the clinical setting. 
TEED was calculated using the formula [(Voltage ${ }^{2} \times$ Frequency $\times$ Pulse width)/Impedance] after conversion of the stimulation current to voltage using individual contact impedances (16).

Speech assessment consisted of a 60-second monologue, and the Assessment of Intelligibility for Dysarthric Speech computerised version (Speech Intelligibility Test; SIT)[17], assessed OFF-medication at the stimulation efficacy threshold for each PW condition. The SIT requires patients to read a list of sentences. A rater (TG) transcribed the sentences. The SIT score provided is number of words transcribed correctly (SIT\%). Both the 60 -second monologue (MON/42) and SIT sentences (SIT/42) were rated using the perceptual rating scale developed by Darley, Aronson and Brown (1969)[18]. The speech samples were rated on a number of speech parameters (out of 7), namely Articulation, Respiration, Resonance, Phonation, Prosody and Rate, giving a composite score out of 42 .

\section{Statistics}

Normality of data and homogeneity of variances between the two conditions were verified using the Shapiro-Wilk and Bartlett tests respectively, with a significance level set to 0.05 . Where data was not normally distributed, the non-parametric Wilcoxon signed rank test was used. $R$ software version 3.4.1 was used for statistical analysis.

\section{RESULTS}

The mean disease duration was $14 \pm 4$ years, and pre-operative MDS-UPDRS-III scores were $42 \pm 6$ OFF and $15 \pm 6$ ON medication. The post operative median MDS-UPDRS rigidity score OFF stimulation (UPDRS item 3.3) was 2 [range 0-3], with two patients not having any clinically detectable rigidity at the contralateral wrist (presumably resulting from the persisting stun effect of electrode implantation). Complete rigidity control with stimulation (score 0 ) could be achieved in all patients using the most efficacious contact. Persistent side effects at these contacts for PW60 and PW30 respectively included slurred speech [11;9], facial $[8 ; 9]$ or limb [5; 5] muscle contraction, sensory symptoms [4; 11], gaze deviation or diplopia [3; 2], autonomic symptoms [1; 3] and other [2; 2] which included head discomfort at PW60 and vertigo at PW30.

The efficacy threshold was significantly greater at PW30 $(3.2 \pm 1.1 \mathrm{~mA})$ than at PW60 $(2.2 \pm$ $0.6 \mathrm{~mA})$ as was the side effect threshold at PW30 $(6.7 \pm 2.0 \mathrm{~mA})$ compared to PW60 (3.8 \pm $1.1 \mathrm{~mA}$ ). These are illustrated in figure 1.

Therapeutic windows could be calculated in 28 out of 30 STNs, (due to lack of detectable contralateral wrist rigidity in two). The TW for PW60 was $1.7 \pm 1.1 \mathrm{~mA}$ [median 1.8; range $5.0 \mathrm{~mA}$ ] and for PW30 was $3.8 \pm 1.6 \mathrm{~mA}$ [median 3.5; range $6.3 \mathrm{~mA}$ ], as shown in figure 2 . 
The mean increase in TW at PW30 was 197\% compared to PW60 [t $(27)=10.6, p \leq 0.001, r=$ 0.89].

The total electrical energy delivered (TEED) was not significantly different between PW60 $(55 \pm 41 \mathrm{~J} / \mathrm{s})$ and PW30 (54 $\pm 40 \mathrm{~J} / \mathrm{s})$ at the threshold for efficacy [t $(27)=0.31, p=0.76$ ], as shown in figure 3.

In the secondary analysis comparing data from all contacts of each electrode, 110 TWs could be calculated and these remained significantly higher at PW30 $(3.5 \pm 1.7 \mathrm{~mA})$ than at PW60 $(1.4 \pm 1.0 \mathrm{~mA}) ; \mathrm{t}(109)=16.3, \mathrm{p} \leq 0.001, r=0.84$.

The timed 10-metre walk using settings at the defined efficacy threshold at PW30 $(8.7 \pm 2.3 \mathrm{~s})$ was faster than at PW60 $(9.2 \pm 2.7 \mathrm{~s}) ; \mathrm{p}<0.001$.

The speech intelligibility score for the spoken task (SIT/42) was improved at PW30 (36 \pm 5 ) than at PW60 ( $33 \pm 5$ ); $\mathrm{p}<0.01$, as was the monologue task (MON/42; $35 \pm 5$ for PW30 vs. 32 \pm 5 at PW60; $p=0.02$ ). In particular, articulation and rate subscores were noted to be sensitive to change in PW settings. The SIT\% scores were not significantly different for PW30 $(96.5 \pm 4.1)$ and PW60 (94.7 \pm 8.7$) ; p>0.05$.

\section{DISCUSSION}

Our results show that the therapeutic window of stimulation using a short pulse width setting of $30 \mu \mathrm{s}$ is significantly greater than that at standard PW settings. The magnitude of this increase is greater than two-fold, and is consistent across all contacts of a given electrode. The change in TW was characterised by a relatively greater increase in the side effect threshold than the efficacy threshold at short PW settings compared to those at standard PW. Our findings are consistent with those reported in a smaller series previously, and is the largest cohort to date confirming the relative increase in the TW at PWs shorter that $60 \mu \mathrm{s}(13)$.

Furthermore, we found that energy consumption using short PW settings at the threshold for rigidity control was not significantly different to that at standard settings. This finding is somewhat unexpected with the assumption of requiring a proportional increase in stimulation amplitude, as a change in the voltage has a relatively greater impact on energy used per unit time than PW. However, our data demonstrates that while PW is halved with $30 \mu$ s settings, the stimulation amplitude that defined the efficacy threshold was only $41 \%$ higher than at standard PW, resulting in no significant net difference in energy consumption.

The timed 10-metre walk showed a small but significant improvement at short PW settings. However, given the magnitude of the difference in times between the two PW conditions, more detailed analysis of gait with sensitive objective outcome measures as well as long 
term subjective patient feedback need to be examined to substantiate any clinically significant benefit. Results of perceptual speech intelligibility showed both the spoken (MON/42) and reading tasks (SIT/42) were significantly better at PW30. However, there was no significant difference in speech intelligibility as measured by the SIT\% score; this may be due to the only mild speech deficits of the patients included in the study. While the cohort of patients examined was not selected on the basis of speech impairment, this finding merits further scrutiny in patients with more profound stimulation induced dysarthria, who may potentially benefit more from short PW settings.

While therapeutic and adverse effects produced by stimulation delivered through a contact are inextricably linked to the precise location of the contact in the brain, global patterns emerging from systematic study of changes in parameter settings are useful despite the confounding factors of natural anatomical variation, variations in surgical technique and precision of targeting. Short PW settings may therefore have beneficial clinical applications in PD patients who have STN DBS. While it may not be relevant to those on standard settings who have an adequate TW, many patients require an increasing level of stimulation over time to control progressive PD symptoms, which may exceed the side effect threshold. Indeed, some patients are found to have a very narrow or even negative TW early in the course of DBS therapy. In these cases, short PW settings can provide an alternative that results in adequate control of motor symptoms while avoiding side effects such as speech and gait dysfunction.

The exact mechanism by which short PW stimulation results in different thresholds for efficacy and adverse effects in STN DBS remains yet to be fully elucidated, but from work done in patients with essential tremor (ET), there are suggestions of more selective stimulation of neural fibre tracts responsible for relief of symptoms while avoiding those responsible for adverse effects. In ET patients, it was calculated that the chronaxie (measure of excitability of neural elements) for suppression of tremor was significantly different to that for induction of ataxia, with values of $27 \mu$ s and $52 \mu$ s respectively. While the authors were not able to stimulate at pulse widths shorter than $60 \mu \mathrm{s}$, they were able to conclude that a stimulation pulse width closer to the chronaxie of tremor suppression would provide a wider therapeutic window between tremor relief and induction of side effects such as ataxia (15). Following this, Reich et al used model derived strength-duration curves for STN stimulation in PD patients to demonstrate a widening difference in the action potential initiation thresholds between small diameter fibres close to the electrode and larger diameter fibres further away, thought to correspond to therapeutic effects and adverse effects respectively (14).

Limitations of our study include non-blinded clinical assessment of rigidity and side effects, assessment early in the post-operative course, and the lack of comprehensive motor assessment and long term follow up on the two conditions. We also only compared PW at $30 \mu \mathrm{s}$, and intermediate PW settings of $40 \mu \mathrm{s}$ and $50 \mu$ s need to be explored as they may have 
a role in cases where $30 \mu$ s settings may not be well-tolerated or as efficacious for any reason. Given the possible underlying mechanistic notion of various PWs resulting in selective stimulation of different neural elements, it may indeed be that the optimal PW lies somewhere in this range, perhaps depending on the clinical variable being optimised.

While a significant increase in the TW of DBS therapy is a welcome finding, randomised double blinded trials with longer term follow up in patients who suffer from stimulationinduced side effects are now needed to confirm the sustained efficacy, tolerability, and thereby clinical application and incorporation into DBS programming paradigms, of short pulse width neurostimulation in PD patients.

\section{Declaration of interests}

No financial or material support was received for this work.

PL has received honoraria from Boston Scientific and Medtronic. TF has received honoraria from Profile Pharma, BIAL, AbbVie, Genus, Medtronic, and St Jude Medical. All other authors declare no conflicts of interest.

\section{REFERENCES:}

1. Limousin, P., Pollak, P., Benazzouz, A., Hoffmann, D., Le Bas, J., Perret, J., Benabid, A. and Broussolle, E. (1995). Effect on parkinsonian signs and symptoms of bilateral subthalamic nucleus stimulation. The Lancet, 345(8942), pp.91-95.

2. Limousin P, Krack P, Pollak P, Benazzouz A, Ardouin C, Hoffmann D, Benabid, A. (1998). Electrical Stimulation of the Subthalamic Nucleus in Advanced Parkinson's Disease. New England Journal of Medicine, 339(16), pp.1105-1111.

3. Weaver, Frances M (2009) Bilateral Deep Brain Stimulation Vs Best Medical Therapy for Patients with Advanced Parkinson Disease: A Randomized Controlled Trial. JAMA 301.1: 63.

4. Kleiner-Fisman, G., Herzog, J., Fisman, D., Tamma, F., Lyons, K., Pahwa, R., Lang, A. and Deuschl, G. (2006). Subthalamic nucleus deep brain stimulation: Summary and meta-analysis of outcomes. Movement Disorders, 21(S14), pp.S290-S304.

5. Tommasi G, Krack P, Fraix V, Le Bas J, Chabardes S, Benabid A, \& Pollak P (2008) Pyramidal tract side effects induced by deep brain stimulation of the subthalamic nucleus. J Neurol Neurosurg Psychiatry, 79, 813-819.

6. Baizabal-Carvallo JF, Jankovic J (2016) Movement disorders induced by deep brain stimulation. Parkinsonism Relat Disord, 25, 1-9. 
7. Tornqvist AL, Schal'en L, Rehncrona S (2004) Effects of different electrical parameter settings on the intelligibility of speech in patients with Parkinson's disease treated with subthalamic deep brain stimulation. Mov Disord, 20, 416-423.

8. Tripoliti E, Zrinzo L, Martinez-Torres I, Frost E, Pinto S, Foltynie T, Holl E, Petersen E, Roughton M, Hariz MI, Limousin P (2010) Effects Of subthalamic stimulation on speech of consecutive patients with Parkinson disease. Neurology, 76, 80-86.

9. Deuschl, G., Herzog, J., Kleiner-Fisman, G., Kubu, C., Lozano, A., Lyons, K., RodriguezOroz, M., Tamma, F., Tröster, A., Vitek, J., Volkmann, J. and Voon, V. (2006). Deep brain stimulation: Postoperative issues. Movement Disorders, 21(S14), pp.S219-S237.

10. Rizzone M. Deep brain stimulation of the subthalamic nucleus in Parkinson's disease: effects of variation in stimulation parameters (2001) Journal of Neurology, Neurosurgery \& Psychiatry. 71(2):215-219.

11. Moro, E., Esselink, R., Xie, J., Hommel, M., Benabid, A. and Pollak, P. (2002). The impact on Parkinson's disease of electrical parameter settings in STN stimulation. Neurology, 59(5), pp.706-713.

12. Dayal, V., Limousin, P. and Foltynie, T. (2017). Subthalamic Nucleus Deep Brain Stimulation in Parkinson's Disease: The Effect of Varying Stimulation Parameters. Journal of Parkinson's Disease, 7(2), pp.235-245.

13. Volkmann J, Moro E, Pahwa R. Basic algorithms for the programming of deep brain stimulation in Parkinson's disease. Mov Disord 2006;21(suppl 14):S284-S289.

14. Reich, M., Steigerwald, F., Sawalhe, A., Reese, R., Gunalan, K., Johannes, S., Nickl, R., Matthies, C., Mclntyre, C. and Volkmann, J. (2015). Short pulse width widens the therapeutic window of subthalamic neurostimulation. Annals of Clinical and Translational Neurology, 2(4), pp.427-432.

15. Groppa S, Herzog J, Falk D, Riedel C, Deuschl G. \& Volkmann J (2013) Physiological and anatomical decomposition of subthalamic neurostimulation effects in essential tremor. Brain, 137, 109-121.

16. Koss, A., Alterman, R., Tagliati, M. and Shils, J. (2005). Calculating total electrical energy delivered by deep brain stimulation systems. Annals of Neurology, 58(1), pp.168-168.

17. Yorkston, K, Beukelman D. (1984) Assessment of Intelligibility of Dysarthric Speech. Austin: Pro-ed, CC Publications. 


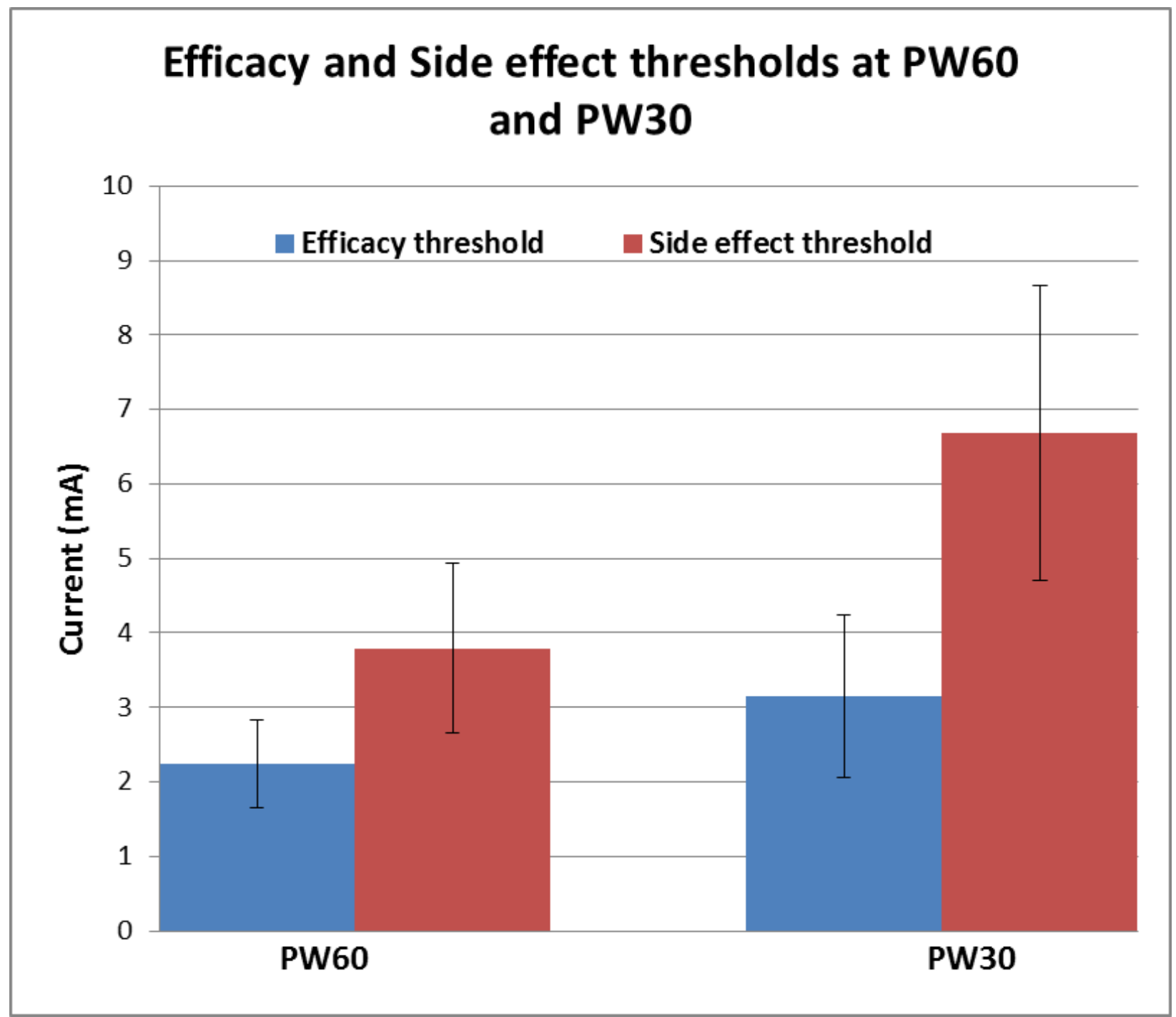

Figure 1: Mean efficacy and side effect thresholds at PW60 and PW30. 


\section{Therapeutic window at 60 and 30 microseconds}

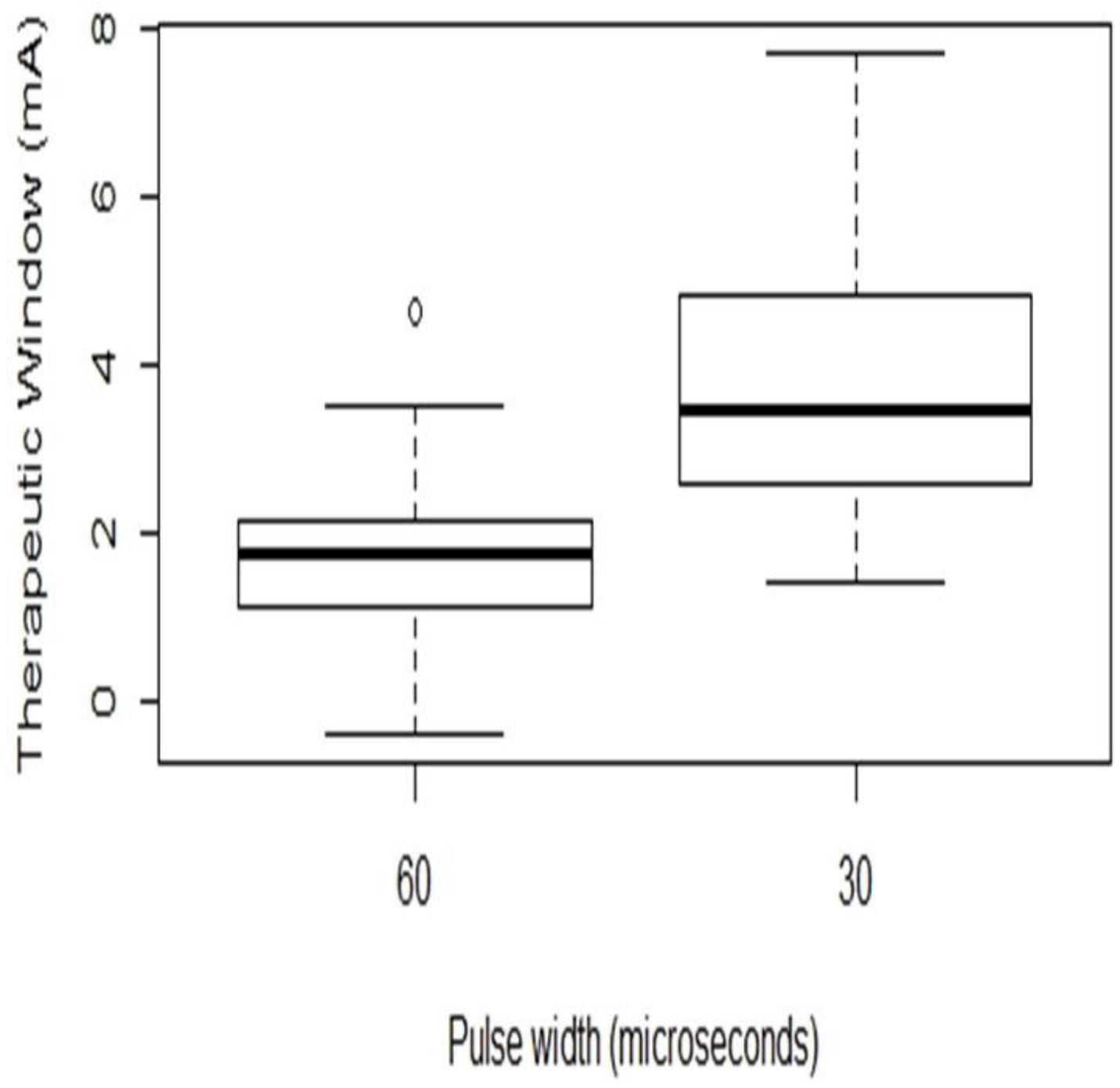

Figure 2: The therapeutic window (difference between side effect and efficacy thresholds) for pulse widths at 60 and 30 microseconds. 


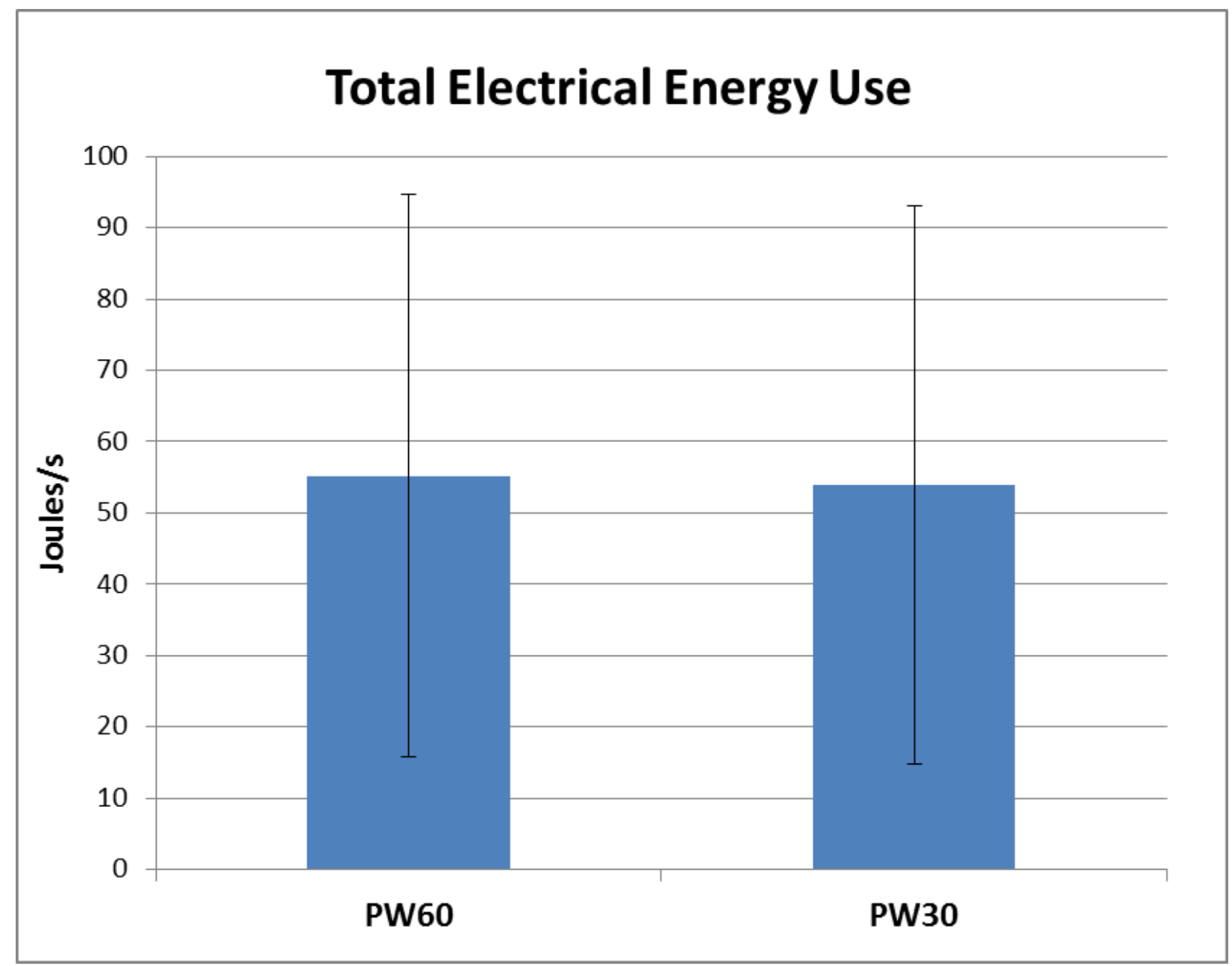

Figure 3: Mean total electrical energy use in Joules per second at PW60 and PW30 at the efficacy thresholds. 